\title{
The role of the TNFa-mediated astrocyte signaling pathway in epilepsy
}

\author{
Rui Chen ${ }^{1}$, Guofang Xue ${ }^{2^{*}}$ (i) and Christian Hölscher ${ }^{2}$
}

\begin{abstract}
Epilepsy is a common disease in the central nervous system. There is growing evidence that epilepsy is associated with glial cells, including astrocytes. Tumor necrosis factor a (TNFa) is a "master regulator" of proinflammatory cytokine production and is secreted by microglia and astrocytes. TNFa secreted by microglia can activate astrocytes. Additionally, TNFa can regulate neuron activity and induce epilepsy by increasing the glutamate release, reducing the expression of $y$-aminobutyric acid, inducing neuroinflammation and affecting the synaptic function in astrocytes. This review summarizes the signaling pathways and receptors of TNFa acting on astrocytes that are related to epilepsy and provides insights into the potential therapeutic strategies of epilepsy for clinical practice.
\end{abstract}

Keywords: Tumor necrosis factor alpha, Astrocytes, Inflammation, Epilepsy

\section{Background}

Epilepsy has been identified as a clinical syndrome since the invention of electroencephalogram in 1929 [1], and causes a great burden on patients and their families. Epilepsy is a common disease of the central nervous system, but the pathogenesis of epilepsy has not been fully clarified [2]. It is currently known that the main pathogenesis of epilepsy is the imbalance of excitatory and inhibitory neurotransmission, which involves altered gene expression, inflammation, oxidative stress, among other factors [2-4]. The increased pro-inflammatory factors can lead to excessive neuronal excitation, which in turn affects the glutamate and $\gamma$-aminobutyric acid (GABA) receptors. Oxidative stress can affect changes in calcium currents and increase the neuronal excitability [2]. Changes in the blood-brain barrier (BBB) are also associated with altered excitability [4].

Astrocytes account for $\sim 30 \%$ of the cells in the human central nervous system (CNS) [5]. Astrocytic inflammation plays a role in numerous neurological diseases,

*Correspondence: xueguofangty@163.com

${ }^{2}$ Neurology Department, The Second Affiliated Hospital of Shanxi Medical University, Taiyuan 030001, Shanxi, China

Full list of author information is available at the end of the article including Alzheimer's disease, ischemic stroke, and epilepsy [6]. Astrocytes not only have neuroprotective functions, but may also destroy nerve cells [7]. Studies using human brain tissues have found that epilepsy is associated with astrocytic and microglial activation [8]. Activation of astrocytes can be seen in the pilocarpine model of temporal lobe epilepsy (TLE) [9], and astrocytes play critical roles in epilepsy. Astrocytes are sensitive to neuronal activity and extracellular changes, protecting neurons from excessive activity and damage [10]. Astrocytes can mediate neuronal activity through ion channels, transmitter receptors and transporters [6]. In a study using two mouse injury models, the cerebral artery occlusion (MCAO) model of ischemic stroke and a neuroinflammation model induced by intraperitoneal injection of $5 \mathrm{mg} / \mathrm{kg}$ lipopolysaccharide (LPS), the reactive astrocytes display two phenotypes, $\mathrm{A}_{1}$ (MCAO phenotype) and $\mathrm{A}_{2}$ (LPS phenotype). This suggests that the transformation of astrocytes to a particular phenotype depends on the nature of the activating stimulus [11]. A more recent study showed that the classically activated neuroinflammatory microglia can induce the $A_{1}$ subtype of reactive astrocytes, through secreting TNF $\alpha$, IL- $1 \alpha$ and C1q. Quantitative polymerase chain reaction also verified that TNF $\alpha$-, IL-1 $\alpha$ - and C1q-knockout mice original author(s) and the source, provide a link to the Creative Commons licence, and indicate if changes were made. The images or other third party material in this article are included in the article's Creative Commons licence, unless indicated otherwise in a credit line to the material. If material is not included in the article's Creative Commons licence and your intended use is not permitted by statutory regulation or exceeds the permitted use, you will need to obtain permission directly from the copyright holder. To view a copy of this licence, visit http://creativecommons.org/licenses/by/4.0/. 
can produce activated microglia, but with a drastically reduced percentage of the $A_{1}$ phenotype of astrocytes [7]. The $A_{1}$ phenotype of astrocytes destroy synapses, while the $\mathrm{A}_{2}$ phenotype repairs the $\mathrm{BBB}$ and protects the CNS from damage induced by the immune response [11]. The $A_{1}$ phenotype has up-regulation of synaptic disruption genes, while the $A_{2}$ phenotype has up-regulation of neurotrophic factors. Although the $A_{1}$ phenotype possesses neurotoxic properties that destroy neurons, the $\mathrm{BBB}$ largely protects the CNS from the neurotoxic damage [5, 7].

Adjacent astrocytes have gap junctions composed of Cx43. The gap junctions enhance cell-to-cell communication with ion coupling and metabolite coupling [12]. In addition, astrocytes can cause neurological damage by changing neurotransmitters, transporters and gap junctions [13]. Extensive experimental evidence has suggested that the gap junctions in astrocytes play a key role in epilepsy. Cx43 has been reported to be upregulated during epilepsy. The Cx43 expression can improve the impaired gap junctions in the kainic acid-induced epilepsy model [14].

In TLE, connexins, potassium channels and water channels are affected [13]. Changes in the levels of astrocyte glutamate transporter-1 (GLT1) and aquaporin-4 have been observed in another kainic acid-induced epilepsy model by real-time polymerase chain reaction, Western blotting, and immunohistochemistry [15].

In astrocytes, the enzyme glutamine synthetase (GS) converts glutamate to glutamine as a precursor of GABA. As a result, defects in GS may increase the neuronal excitability due to the increased extracellular glutamate concentration, which is positively correlated neuronal excitability [13]. At the same time, studies using Genetic Absence Epilepsy Rats from Strasbourg (GAERS), Wistar Albino Glaxo Rats from Rijswijk (WAG/Rij) and control Wistar animals have proven that epilepsy is related to the imbalance between GABA and glutamate and the up-regulation of glial fibrillary acidic protein (GFAP), an astrocytic marker [16].

The astrocytic TNF $\alpha$ participates in the pathological process of epilepsy [17]. TNF $\alpha$, initially recognized as a target for treatment of tumors, has been confirmed as a target of inflammation [18]. The TNF $\alpha$-mediated inflammation is a key element in seizures. TNF $\alpha$ not only causes damage to the BBB, but also affects the CNS immune function through multiple pathways to produce inflammatory factors $[18,19]$. Hence, it is essential to explore the role of TNF $\alpha$ in epilepsy neuropathology.

\section{TNFa}

TNF $\alpha$ is an inflammatory cytokine that plays an important role in the pathogenesis of CNS diseases. TNF $\alpha$ is a trimer composed of three monomers with a relative molecular mass of $17 \times 10^{3}[20,21]$. TNF $\alpha$ gene is located within the major histocompatibility complex class II region, and its expression is maintain at homeostasis [22]. TNF $\alpha$ plays an important role in the CNS (Table 1). Under physiological conditions, TNF $\alpha$ promotes cell proliferation, participates in cognitive processes, and controls ion homeostasis. Under pathological conditions, TNF $\alpha$ causes neurotoxicity, promotes proliferation of glial cells and regulates $\mathrm{T}$ cell development $[26,27]$. TNF $\alpha$ is involved in epilepsy through releasing glutamate, mediating the downregulation of connexin 43 and controlling synaptic transmission [12, 20, 23, 28, 29]. TNF $\alpha$-treated mice show an increased level of glutaminase protein and improved glutamate production [30]. TNF $\alpha$ also participates in the epileptic process by inducing changes in neuronal excitability via increased glutamate receptors and enhanced GABA receptor endocytosis [31].

There are two types of TNF $\alpha$ receptors in the CNS, namely TNFR1 and TNFR2. They are involved in different signaling pathways and have different functions. TNFR1 plays a role in regulating glutamate receptor transport, while TNFR2 plays a neuroprotective role [32, 33].

The TNF $\alpha$ levels are significantly increased in the hippocampus infected with Theiler's murine encephalomyelitis virus (TMEV). In the TMEV-induced epilepsy, the ratio of TNFR1 to TNFR2 in the hippocampus is increased [19], suggesting that TNF $\alpha$ and TNFR1 are involved in epilepsy. The TNF $\alpha$-mediated inflammation leads to seizures in mouse epilepsy models via increased TNF $\alpha$ and TNFR1 expression [32]. TNF $\alpha$ increases glutamate release after binding to TNFR1, resulting in the increase of excitatory synaptic transmission [19]. In addition, after the activation of the TNF $\alpha$-TNFR1 signaling pathway, a variety of adaptor proteins can activate transcription factors to affect cell growth, cell death and inflammation [20]. In conclusion, selective inhibition of TNF $\alpha$ and TNFR1 can reduce the susceptibility to epilepsy [19]. TAK1 (transforming growth factor- $\beta$-activated kinase 1) and IKK $\beta$ (inhibitor of $\kappa B$ (ІкB) kinase $\beta$ ) in the

Table 1 Roles of TNFa in epilepsy

\begin{tabular}{ll}
\hline Function & Refs \\
\hline Promote hyperexcitability via purinogenic signaling, glutamate & {$[23]$} \\
release and modulation of neuronal transmitter release & \\
Increase ictal discharges & {$[24]$} \\
Modulate glutamate receptor transport & {$[19]$} \\
Induce apoptosis & {$[25]$} \\
\hline
\end{tabular}


scaffolding ubiquitin network constructed by the TNFR signaling can activate mitogen-activated protein kinase (MAPK) and nuclear factor $\mathrm{KB}$ (NF-kB), respectively [18], indicating that MAPK and $\mathrm{NFKB}_{\mathrm{K}}$ also play a role in the TNF-TNFR axis.

\section{Astrocytes and reactive astrocytes}

Astrocytes are the most abundant glial cells in the CNS. Astrocytes have long and branched processes. The morphology of an astrocyte is related to the expression of glial proteins and neuronal neuroglobulin. It has been found that astrocytes possess structural plasticity. Glutamate, action potential, mGluRs and IkkB /NF- $\mathrm{kB}$ mediate structural remodeling of astrocytes [34]. Astrocyte morphology is responsible for normal brain functions such as neurogenesis, synaptic formation and neurotransmitter transmission [5].

IL-1 $\alpha$, TNF $\alpha$ and C1q secreted by microglia can act on astrocytes and induce their switch to the $A_{1}$ reactive phenotype. Reactive astrocytes differ from non-reactive astrocytes in their morphology. The reactive astrocytes are characterized by hypertrophy of cells and processes that is positively correlated with injury severity in reactive astrocytes, and increased GFAP expression [7, 35].

The morphological and structural differences between A1 reactive and non-reactive astrocytes suggest distinct functions of the two types of astrocyte. The A1 reactive astrocytes are neurotoxic. They can impair normal brain functions, they produce pro-inflammatory chemokines and cytokines resulting in neuroinflammation, and impairs synaptic function and phagocytosis. The A1 reactive astrocytes also increase the level of neuroexcitatory glutamate and inhibit the effects of GABA, thereby promoting the development of epilepsy [35].

\section{TNFa signaling pathway in astrocytes \\ $G A B A$ receptors and glutamate receptors}

One possible mechanism of epilepsy generation is the imbalance between the excitatory glutamate and the inhibitory GABA neurotransmitter. In excitatory neurons, glutamate released at synapses can promote epilepsy, while in inhibitory neurons, glutamate decarboxylase converts glutamate to GABA and suppresses epilepsy [3].

Astrocytes may be involved in seizure generation via their release of TNF $\alpha$ alone or in combination with glutamate. TNF $\alpha$ controls the neurotransmission of glutamate and GABA in post-synaptic membranes. In addition, blocking the release of GABA precursor glutamine from astrocytes, or the loss of glutamine synthetase, can result in increased synaptic glutamate levels, decreased GABA synthesis and excessive neuronal excitement $[3,13]$.
GABA transporters GAT- 1 and GAT-3 can regulate the level of extracellular GABA. Furthermore, in the MCAO model, the participation of GABAergic transmission in post-ischemia epilepsy has been determined by measuring GAT- 1 and GAT-3 protein levels at these sites. TNF $\alpha$ released during epilepsy can enhance GAT-1 and GAT-3 expression and reduce GABA levels, which in turn aggravate epilepsy [36].

Studies in the pilocarpine-induced mouse model have found that the epilepsy frequency correlates with the loss of inhibitory GABAergic neurons. Astrocyte dysfunction increases glutamate levels and greatly reduces the $\mathrm{Cl}^{-}$ flow into the extracellular space, inducing depolarization. As $\mathrm{Cl}^{-}$gradient has been shown to control the inhibitory effect of $\mathrm{GABA}_{\mathrm{A}}$ receptors, $\mathrm{GABA}_{\mathrm{A}}$ receptor function may be reduced by the decreased activity of the associated $\mathrm{Cl}^{-}$channel $[3,13]$.

Interestingly, studies in the GAERS, WAG/Rij and Wistar animal models have shown that the increased GABA uptake by astrocytes significantly promotes the excitatory glutamate levels in somatosensory cortex and thalamic nuclei, leading to excessive neuronal excitement [16].

The balance between glutamate production and elimination ensures the maintenance of a normal level of glutamate. Astrocytes express transporters such as glutamate-aspartate transporter (GLAST) and GLT1, which are essential for glutamate clearance [37]. The uptake of glutamate in the synaptic cleft is important for the maintenance of physiological concentrations of glutamate and thus homeostasis. TNF $\alpha$ up-regulates the glutaminase activity to promote the production while reducing the elimination of glutamic acid, finally leading to glutamic acid accumulation [38]. Moreover, when astrocytes transform into the reactive phenotype, they express a lowered level of GLAST and GLT1, which may also lead to glutamate accumulation [37]. When glutamate reaches pathological levels, it can have toxic effects and cause damage to synapses [38]. Zhou et al. have found that TNF $\alpha$ inhibits glutamate uptake through the NF-kB signaling pathway and enhances the phagocytosis of astrocytes in human NCRM-1 astrocytes [39]. In recent years, autoimmune encephalitis, which is associated with epileptic seizures, has been studied at a greater level. Interestingly, $N$-methyl- $d$-aspartate (NMDA) receptor (NMDAR), the postsynaptic ionotropic glutamate receptor, is one of the pathogenic factors for this autoimmune disease [37].

\section{AMPA receptors}

The AMPA receptor is a glutamate-gated ion channel that regulates excitatory synaptic transmission and maintains a balance between excitatory and inhibitory synapses [40]. AMPA receptor ubiquitination maintains a 
balance of excitability and inhibition in neurons [41]. The AMPA receptors dynamically regulate the synaptic plasticity via their altered amount, subunit composition and biological characteristics in the post-synaptic membrane [40]. The AMPA receptor function is mediated by helper proteins and transmembrane AMPA receptor regulatory proteins (TARPs). TARP 8 , located in the forebrain, affects the kinetics of AMPA receptor and prolongs the receptor activation time, which is associated with TLE [42]. The structural abnormalities and dysfunction of AMPA receptors can enhance neuronal excitability, contributing to the generation of epilepsy [43].

AMPA receptors are highly expressed on astrocytes and neurons, and TNF $\alpha$ can enhance excitotoxicity through the AMPA receptors $[6,20]$. The AMPA receptors contain four subunits, including GluR1 to GluR4. Enhanced expression of GluR1 flip variants in astrocytes has been detected in the hippocampi of epilepsy animal models. This suggests that astrocytes may induce epilepsy through the AMPA receptors [6]. Abnormal palmitylation of AMPA receptors enhances epilepsy susceptibility, in which the GluA1 subunit plays a key role. Deficiency of GluA1 palmitylation disrupts the balance of excitatory and inhibitory synapses without affecting THE brain structure [44].

One study has confirmed that parallel fibers (PFs) can activate AMPA receptors on Bergmann glia (BG), a type of astrocyte, to induce calcium ion changes, thereby releasing glutamate. Overactivation of AMPA receptors expressed by neurons can also induce neurotoxicity. In addition, studies have found that higher concentrations of TNF $\alpha$ can cause damage by activating the $\mathrm{Ca}^{2+}$-permeable AMPA receptors, altering synapse transmission and producing excitotoxicity [29]. The combination of TNF $\alpha$ and TNFR1 alters AMPA receptors in the synapse, increasing neuronal excitability, while TNFR2 plays a protective role [32]. TNF $\alpha$ readjusts glutamatergic neurotransmission by regulating AMPA receptors, thereby controlling the synaptic function. In conclusion, TNF $\alpha$ performs synaptic regulation and excitability by controlling AMPA receptor trafficking [20].

AMPA receptors act as a target for epilepsy therapy. Currently, AMPA receptor antagonist has been found to have anticonvulsant effects, reducing the excitatory postsynaptic current frequency and inhibiting the excitatory synaptic transmission in neurons, resulting in decreased spontaneous epileptic-like discharge in brain tissue samples from children [45]. Currently known AMPA receptor antagonists that can be used to treat epilepsy are perampanel and talampanel [43]. Perampanel is used for partial-onset and tonic-clonic seizures [46, 47]. However, talampanel is not recommended for use in the clinical treatment of epilepsy due to adverse reactions [48].

\section{NF-KB signaling pathway}

The NF- $k B$ signaling pathway is a key pathway of the central inflammatory response. It induces the expression of many pro-inflammatory factors, such as cell adhesion factors, cytokines and iNOS [49]. Astrocytes, after prolonged exposure to glutamic acid, activate the NF- $\mathrm{kB}$ signaling pathway via NMDA receptors and the activation of I-type voltage-sensitive calcium channel. The activated NF- $\mathrm{kB}$ can be involved in structural and synaptic plasticity. One study demonstrated that the activated NF- $\mathrm{KB}$ can activate glutamatergic neurons and promote excitatory synaptic formation. In addition, NF- $\mathrm{kB}$ regulatory sites have been found on GLT-1 gene [50].

TNF $\alpha$ promotes inflammation through the NF- $\kappa B$ signaling pathway [51]. Previous research demonstrated that NF- $\mathrm{KB}$ plays a role in determining the cell fate [52]. TNF $\alpha$ disrupts the BBB, causing excessive neuronal excitement, and thus epilepsy in the pilocarpine-induced status epilepticus rat model. Fluoro-Jade B staining and immunohistochemical analysis of TNF $\alpha$ and NF- $\mathrm{kB}$ subunits have demonstrated upregulation of the TNF p55 receptor (TNFR1) and TNF p75 receptor (TNFR2) in astrocytes [53]. Anti-TNF biologics can prevent TNF $\alpha$ from binding to receptors TNFR1 and TNFR2 via the NF- $\mathrm{KB}$ signaling, which reduces inflammation. Therefore, TNF $\alpha$ induces apoptosis by NF- $\mathrm{kB}$ signaling and aggravates inflammation. Experiments have shown the existence of an NF-kB-independent cell death checkpoint that limits apoptosis by inactivation of the receptor-interacting serine-threonine kinase 1 (RIPK1). Disruption of this checkpoint could cause inflammation in vivo. Moreover, blocking RIPK1 can inhibit the TNF $\alpha$-mediated inflammatory processes [51].

The TNF $\alpha$-mediated phosphorylation of NF-kB subunits causes angioedema with neutrophil infiltration and astrocyte damage [53]. At the same time, the TNF $\alpha-$ NF- $\mathrm{KB}$ signaling also increases the expression of drug transporters, including P-glycoprotein (P-gp) and multidrug resistance-associated protein (MRP). There is evidence that P-gp and MRP are present in astrocyte processes and in primary cultures of rat astrocytes. Astrocytes increase P-gp and MRP expression via the $\mathrm{TNF} \alpha-\mathrm{NF}-\mathrm{kB}$ signaling. This may be one of the mechanisms of refractory epilepsy [17].

Moreover, NF- $\mathrm{KB}$ has been shown to regulate the expression of COX-2. COX-2 can shorten the interval of seizures, leading to repeated seizures. The peptide $\kappa B$-decoy binds to free NF- $\kappa B$ and affects its transcription. Experimental results have shown that $\kappa \mathrm{B}$-decoy could inhibit NF- $\mathrm{kB}$ activation in epileptic-related brain regions and decrease the mRNA and protein expression of COX-2, thus reducing seizures [54]. 


\section{Signal transducer and activator of transcription 3 (STAT3) signaling pathway}

STAT3 is a transcription factor that can cause cell proliferation, differentiation and apoptosis. Reactive astrocytes can repair lesions and inhibit inflammation through the STAT3 signaling pathway, in contrast to the NF- $k B$ signaling pathway [35]. However, in the $A \beta$-oligomer-induced AD model, STAT3 activation can mediate astrocyte activation, resulting in neurotoxicity [55]. Moreover, the STAT3 signaling pathway has also been shown to promote neuronal death, which is associated with epilepsy.

STAT3 polymorphism is associated with the prevalence of epilepsy in children. The levels of STAT3 and p-STAT3 increase in brain tissues of children with epilepsy, as detected by immunohistochemistry. Previous studies have shown high expression of p-STAT3 in the hippocampus in a rat epilepsy model [56]. Another study showed that STAT3 expression is prominent in glial cells and neurons in epilepsy. Moreover, in the kainic acid (KA)-induced TLE epileptic model, the long noncoding RNA urothelial cancer-associated 1 can inhibit astrocyte activation through the JAK/STAT signaling pathway, improving the adverse effects of epileptic episodes. This suggests that the STAT signaling pathway participates in astrocyte activation and plays an important role in the induction of epilepsy [57]. Similarly, miR-21-5p can inhibit STAT3 expression and reduce neuronal apoptosis and damage in the hippocampus, thus exerting protective effects on neurons of epileptic rats [58]. STAT3 phosphorylation is associated with secretion of neuroinflammatory cytokines, such as IL-6, IL- $1 \beta$ and TNF- $\alpha$. Thus, inhibition of STAT3 is also effective in suppressing neuroinflammation [59].

The STAT3 signaling pathway is also crucial for astrocyte development. TNF $\alpha$ promotes the differentiation of neural progenitor cells into astrocytes through induction of STAT3 activation, as revealed by Western blot analysis of total cell lysates. Immunocytochemical staining also demonstrated that the inhibition of STAT3 can inhibit astrocytic differentiation and increase neuronal differentiation of neural progenitor cells under inflammatory conditions. In addition, the application of STAT3targeting siRNA (siSTAT3) decreased the TNF $\alpha$-induced STAT3 activation, inhibiting astrocyte activation [60].

TNF $\alpha$ is also able to enhance the expression of kidney type glutaminase (KGA) via the STAT3 signaling pathway in astrocytes, catalyzing the hydrolysis of glutamine to glutamate and ammonia. The induction of KGA expression by TNF $\alpha$ is inhibited by selective inhibition of STAT3 with a STAT3 inhibitor STA-21. One study demonstrated that the TNF $\alpha$-mediated KGA expression is associated with increased phosphorylation of STAT3 [28]. Furthermore, the increased KGA expression in astrocytes will increase glutamate content. Astrocytes take up glutamate, thereby reducing GABA synthesis and increasing neuronal excitability, finally decreasing the threshold of seizure induction [31]. The TNF $\alpha-S T A T 3$ pathway not only activates astrocytes but also enhances astrocyte uptake of glutamate.

Dasatinib is an FDA-approved drug for the treatment of chronic myeloid leukemia. In an LPS-induced neuroinflammation model, dasatinib inhibits microglial and astrocytic activation via the AKT/STAT3 signaling pathway and leads to a decreased level of proinflammatory cytokines [61].

\section{MAPK signaling pathway}

MAPK signaling pathways include the extracellular signal regulated kinase (ERK) pathway, the p38 pathway and the C-Jun $\mathrm{N}$-terminal kinases (JNK) pathway. Each pathway plays a different role in epilepsy. The ERK/ MAPK signaling pathway up-regulates NMDA receptors and enhances neuronal excitability [62]. There is growing evidence that the p38 MAPK signaling pathway is associated with cognitive impairment in epilepsy. In the rat model of PTZ-induced epilepsy, the p38 MAPK signaling pathway modulates apoptosis and impairs cognitive function [63]. In addition, ferrostatin 1 alleviates the KA-induced cognitive impairment in TLE rats by upregulating the expression of synaptophysin and postsynaptic density protein-95 via the p38 MAPK signaling pathway [64]. The p38 MAPK signaling pathway is associated with refractory epilepsy. SB202190, an antagonist of p38 MAPK, inhibits epileptic seizures and increases the concentrations of valproate and lamotrigine in rats [65]. Furthermore, SB203580, which inhibits the p38 MAPK signaling pathway in rats, repairs the damaged hippocampal neurons and reduces the frequency of seizure attack by down-regulating the levels of Adenosine A1 receptor and type 1 equilibrative nucleoside transporter [66]. The activation of JNK/MAPK signaling pathway occurs at both acute and chronic stages of epileptic development. It participates in astrocyte activation, neuronal death and mossy fiber sprouting [67]. The MAPK signaling pathway regulates RNA-binding proteins (RBPs), which are responsible for mRNA translation. Dysregulated RBPs may result in expression of epilepticrelated factors. Therefore, the MAPK signaling pathway mediates the translation of synaptic proteins to control synaptic excitability and promote epilepsy development [62].

TNF $\alpha$ can activate the MAPK pathway, which upregulates transcription of pro-inflammatory genes via TNFR1 [51]. Consistently, TNF $\alpha$ stimulates astrocytes, leading to activation of the p38 MAPK pathway and the production of nitric oxide as part of the inflammation pathway [68]. 
In addition, the TNF $\alpha-M A P K$ signaling is related to gap junctions in astrocytes as well [14]. TNF $\alpha$ inhibits the expression of $\mathrm{Cx} 43$ gap junctions in spinal astrocytes and reduces communication between glial cells during inflammation [69]. P38 MAPK participates in Cx43 hemichannel activation and astrocytic inflammatory activation. Application of a p38 MAPK blocker could suppress the ethidium (a fluorescent agent that evaluates cell coupling) signal, which detects hemichannel activity as it enters normal cell cytoplasm through hemichannels, uptake of ethidium by hippocampal astrocytes treated with ethanol, thereby repressing the ethanol-induced channel opening in astrocytes [70]. P38 MAPK activates Cx43 gap junctions in neurons to cause synchrony of firing and participates in the development of epilepsy.

\section{Purinergic signaling}

The important role of astrocyte-neuron conduction in the pathogenesis of epileptogenesis is increasingly recognized. Accumulating evidence suggests that there is an enhanced release of glutamate by astrocytes in TLE models [23]. TNF $\alpha$ not only controls the release of glutamate from astrocytes, but also controls the exocytosis of glutamate. Meanwhile, there is evidence that $\mathrm{Ca}^{2+}$ and purinergic receptors have a bidirectional cross-talk. On the one hand, astrocytes activated by the release of glutamate, adenosine triphosphate (ATP) and cyclic adenosine monophosphate activate the purinergic receptor through $\mathrm{Ca}^{2+}$ waves. On the other hand, ATP released by astrocyte hemichannels activates metabolic P2Y receptors, thereby inducing intracellular $\mathrm{Ca}^{2+}$ release from the endoplasmic reticulum. Correlatively, ionic P2X receptors can close gap junctions. Previous studies have demonstrated that inflammatory cytokines such as TNF $\alpha$ are involved in epileptogenesis via the ATP-purinergic receptors $[14,20,23]$. However, in the absence of TNF $\alpha$, ATPmediated P2Y1 receptors cause elevation of intracellular $\mathrm{Ca}^{2+}$, while glutamate release is reduced. When TNF $\alpha$ concentration increases, TNFR1 causes activation of the P2Y1 receptors and makes astrocyte $\mathrm{Ca}^{2+}$ levels change transitorily. Therefore, selective antagonism of P2Y1 receptors prevents TNF $\alpha$ from triggering astrocyte $\mathrm{Ca}^{2+}$ transients, and concurrently normalizes the enhanced miniature excitatory postsynaptic current (mEPSC) frequencies. Similarly, the P2Y1 receptor-activated transmitters interact with presynaptic NMDA receptors, boosting the mEPSC frequency, thereby increasing synaptic transmitter release. In summary, TNF $\alpha$ participates in the transport of glutamate neurotransmitters in astrocytes via the $\mathrm{Ca}^{2+}$-induced purinergic signaling [23].

\section{Conclusions}

Epilepsy is a chronic brain disease characterized by seizures, transience, repetition and rigidity. The abnormal, highly synchronized discharge of neurons is the pathogenesis factor of epilepsy. Epilepsy is closely related to neuroinflammation. The increase of inflammatory

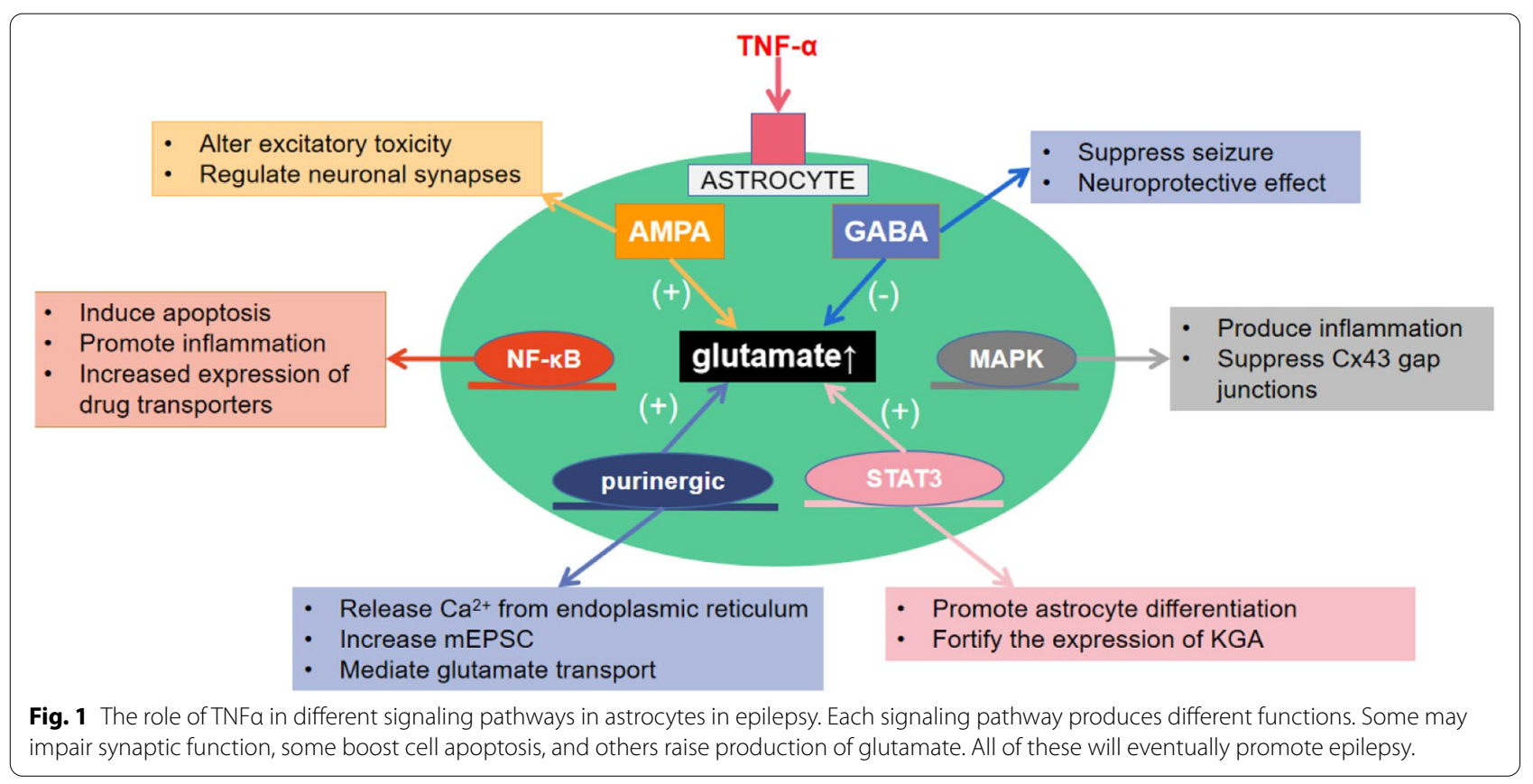


cytokines and cells is a key factor in epileptic foci. IL- $1 \alpha, \mathrm{TNF} \alpha$, and $\mathrm{C} 1 \mathrm{q}$ released by microglia transform astrocytes into the A1 reactive phenotype. Astrocytes are one of the targets of TNFa during epilepsy. In this review we describe an important relationship of TNF $\alpha$ with neuronal excitability in the presence of reactive astrocytes. In astrocytes, TNF $\alpha$ affects glutamate uptake and intercellular gap junctions. TNF $\alpha$ can regulate signaling pathways such as NF- $\mathrm{kB}$, and control receptors such as AMPA receptors (Fig. 1). The former could cause apoptosis to produce epilepsy and the latter could lead to neuronal overexcitation. However, TNF $\alpha$ was rarely studied in epilepsy patients, and it remains unclear which pathway or receptor plays a major role in epilepsy. At the same time, further studies on A1 reactive astrocytes and epilepsy-related signaling pathways are needed. In addition, we need to consider whether all inflammatory mediators and signaling pathways can be used as therapeutic targets for epilepsy. The relationship between NF-kB and STAT3 signaling pathways also requires further experimental exploration.

\begin{abstract}
Abbreviations
TNFa: Tumor necrosis factor a; GABA: $\gamma$-aminobutyric acid; BBB: Blood-brain barrier; MCAO: Middle cerebral artery occlusion; Cx43: Connexin-43; GLT1: Glutamate transporter-1; GS: Glutamine synthetase; GFAP: Glial fibrillary acidic protein; TMEV: Theiler's murine encephalomyelitis virus; MAPK: Mitogenactivated protein kinase; NF-kB: Nuclear factor KB; GAT: Gamma-aminobutyric acid transporter; GLAST: Glutamate-aspartate transporter; NMDA: N-methyld-aspartate; TARPs: Transmembrane AMPA receptor regulatory proteins; PFs: Parallel fibers; BG: Bergmann Glia; iNOS: Inducible nitric oxide synthase; P-gp: P-glycoprotein; MRP: Multidrug resistance-associated protein; COX-2: Cyclooxygenase-2; STAT3: Signal transducer and activator of transcription 3; TLE: Temporal lobe epilepsy; KGA: Kidney type glutaminase; LPS: Lipopolysaccharide; ERK: Extracellular regulated protein kinase; JNK: c-Jun N-terminal kinase; NMDA: N-methyl-D-aspartic acid receptor; RBPs: RNA-binding proteins; ATP: Adenosine triphosphate; mEPSC: Miniature excitatorypostsynaptic current.
\end{abstract}

\section{Acknowledgements}

The authors would like to thank Dr. Holscher of the Neurology Department of Second Affiliated Hospital of Shanxi Medical University on his suggestions on this review.

\section{Authors' contributions}

Guofang Xue conceptualized and revised the article. Rui Chen searched and read the articles related to epilepsy, summarized the views and was a major contributor in writing the manuscript. All authors read and approved the final manuscript.

\section{Funding}

This review was supported by the UCB Foundation of China Association Against Epilepsy (2017007), the National Natural Science Foundation of China (81601038) and the Department of Resource and Social Security of Shanxi Province (Jin fiscal and Social Council [2019] 91st).

Availability of data and materials

Not applicable.

\section{Declarations}

Ethics approval and consent to participate Not applicable.
Consent for publication

Not applicable.

\section{Competing interests}

The authors declare no financial or other conflicts of interest.

\section{Author details}

${ }^{1}$ Shanxi Medical University, Taiyuan 030001, Shanxi, China. ${ }^{2}$ Neurology Department, The Second Affiliated Hospital of Shanxi Medical University, Taiyuan 030001, Shanxi, China.

Received: 21 February 2021 Accepted: 15 September 2021

Published online: 20 October 2021

\section{References}

1. Sontheimer H. Diseases of the Nervous System. Elsevier. 2015:61-95.

2. Yuen AWC, Keezer MR, Sander JW. Epilepsy is a neurological and a systemic disorder. Epilepsy Behav. 2018;78:57-61.

3. Patel DC, Tewari BP, Chaunsali L, Sontheimer H. Neuron-glia interactions in the pathophysiology of epilepsy. Nat Rev Neurosci. 2019;20(5):282-97.

4. Manford M. Recent advances in epilepsy. J Neurol. 2017:264(8):1811-24.

5. Liddelow SA, Barres BA. Reactive Astrocytes: Production, Function, and Therapeutic Potential. Immunity. 2017;46(6):957-67.

6. Siracusa R, Fusco R, Cuzzocrea S. Astrocytes: Role and Functions in Brain Pathologies. Front Pharmacol. 2019;10:1114.

7. Liddelow SA, Guttenplan KA, Clarke LE, Bennett FC, Bohlen CJ, Schirmer $L$, et al. Neurotoxic reactive astrocytes are induced by activated microglia. Nature. 2017;541(7638):481-7.

8. Zurolo E, Iyer A, Maroso M, Carbonell C, Anink JJ, Ravizza T, et al. Activation of toll-like receptor, RAGE and HMGB1 signalling in malformations of cortical development. Brain. 2011;134(4):1015-32.

9. Clasadonte J, Morel L, Barrios-Camacho CM, Chiang MS, Zhang J, lyer $L$, et al. Molecular analysis of acute and chronic reactive astrocytes in the pilocarpine model of temporal lobe epilepsy. Neurobiol Dis. 2016;91:315-25.

10. Burda JE, Bernstein AM, Sofroniew MV. Astrocyte roles in traumatic brain injury. Exp Neurol. 2016;275 Pt 3(0 3):305-15.

11. Zamanian JL, Xu L, Foo LC, Nouri N, Zhou L, Giffard RG, et al. Genomic analysis of reactive astrogliosis. J Neurosci. 2012;32(18):6391-410.

12. Morioka N, Zhang FF, Nakamura Y, Kitamura T, Hisaoka-Nakashima K, Nakata Y. Tumor necrosis factor-mediated downregulation of spinal astrocytic connexin43 leads to increased glutamatergic neurotransmission and neuropathic pain in mice. Brain Behav Immun. 2015;49:293-310.

13. Steinhäuser C, Grunnet M, Carmignoto G. Crucial role of astrocytes in temporal lobe epilepsy. Neuroscience. 2016;323:157-69.

14. Li Q, Li QQ, Jia JN, Liu ZQ, Zhou HH, Mao XY. Targeting gap junction in epilepsy: Perspectives and challenges. Biomed Pharmacother. 2019;109:57-65.

15. Hubbard JA, Szu JI, Yonan JM, Binder DK. Regulation of astrocyte glutamate transporter-1 (GLT1) and aquaporin-4 (AQP4) expression in a model of epilepsy. Exp Neurol. 2016;283(Pt A):85-96.

16. Çavdar S, Kuvvet Y, Sur-Erdem I, Özgür M, Onat F. Relationships between astrocytes and absence epilepsy in rat: An experimental study. Neurosci Lett. 2019;712:134518.

17. Wang $X$, Huang $S$, Jiang $Y$, Liu Y, Song $T$, Li D, et al. Reactive astrocytes increase the expression of P-gp and Mrp1 via TNF- $a$ and NF-KB signaling. Mol Med Rep. 2018;17(1):1198-204.

18. Kalliolias GD, Ivashkiv LB. TNF biology, pathogenic mechanisms and emerging therapeutic strategies. Nat Rev Rheumatol. 2016;12(1):49-62.

19. Patel DC, Wallis G, Dahle EJ, McElroy PB, Thomson KE, Tesi RJ, et al. Hippocampal TNFa Signaling Contributes to Seizure Generation in an Infection-Induced Mouse Model of Limbic Epilepsy. eNeuro. 2017;4(2) ENEURO.0105-17.2017-

20. Santello M, Volterra A. TNFa in synaptic function: switching gears. Trends Neurosci. 2012;35(10):638-47.

21. Rana A, Musto AE. The role of inflammation in the development of epilepsy. J Neuroinflammation. 2018;15(1):144. 
22. Montgomery SL, Bowers WJ. Tumor necrosis factor-alpha and the roles it plays in homeostatic and degenerative processes within the central nervous system. J Neuroimmune Pharmacol. 2012;7(1):42-59.

23. Bedner P, Steinhäuser C. TNFa-Driven Astrocyte Purinergic Signaling during Epileptogenesis. Trends Mol Med. 2019;25(2):70-2.

24. Chong S-A, Balosso S, Vandenplas C, Szczesny G, Hanon E, Claes K, et al. Intrinsic Inflammation Is a Potential Anti-Epileptogenic Target in the Organotypic Hippocampal Slice Model. Neurotherapeutics. 2018;15(2):470-88.

25. Ananias TM, Lília DS-L. Apoptosis through Death Receptors in Temporal Lobe Epilepsy-Associated Hippocampal Sclerosis. Mediat Inflamm. 2016;2016:8290562

26. Decourt B, Lahiri DK, Sabbagh MN. Targeting Tumor Necrosis Factor Alpha for Alzheimer's Disease. Curr Alzheimer Res. 2017;14(4):412-25.

27. Atretkhany KN, Gogoleva VS, Drutskaya MS, Nedospasov SA. Distinct modes of TNF signaling through its two receptors in health and disease. J Leukoc Biol. 2020;107(6):893-905.

28. Milewski K, Bogacińska-Karaś M, Hilgier W, Albrecht J, Zielińska M. TNFa increases STAT3-mediated expression of glutaminase isoform KGA in cultured rat astrocytes. Cytokine. 2019;123:154774.

29. Shim HG, Jang SS, Kim SH, Hwang EM, Min JO, Kim HY, et al. TNF-a increases the intrinsic excitability of cerebellar Purkinje cells through elevating glutamate release in Bergmann Glia. Sci Rep. 2018;8(1):11589.

30. Wang $K$, Ye L, Lu H, Chen H, Zhang Y, Huang Y, et al. TNF-a promotes extracellular vesicle release in mouse astrocytes through glutaminase. J Neuroinflammation. 2017;14(1):87.

31. Vezzani A, Friedman A, Dingledine RJ. The role of inflammation in epileptogenesis. Neuropharmacology. 2013;69:16-24.

32. Chen YC, Zhu GY, Wang X, Shi L, Jiang Y, Zhang $X$, et al. Deep brain stimulation of the anterior nucleus of the thalamus reverses the gene expression of cytokines and their receptors as well as neuronal degeneration in epileptic rats. Brain Res. 2017;1657:304-11.

33. Ortí-Casañ N, Wu Y, Naudé PJW, De Deyn PP, Zuhorn IS, Eisel ULM. Targeting TNFR2 as a Novel Therapeutic Strategy for Alzheimer's Disease. Front Neurosci. 2019;13:49.

34. Zhou B, Zuo YX, Jiang RT. Astrocyte morphology: Diversity, plasticity, and role in neurological diseases. CNS Neurosci Therapeutics. 2019;25(6):665-73.

35. Li X, Li M, Tian L, Chen J, Liu R, Ning B. Reactive Astrogliosis: Implications in Spinal Cord Injury Progression and Therapy. Oxidative Med Cell Longev. 2020;2020:9494352.

36. Zhang W, Wang L, Pang X, Zhang J, Guan Y. Role of microRNA-155 in modifying neuroinflammation and $\gamma$-aminobutyric acid transporters in specific central regions after post-ischaemic seizures. J Cell Mol Med. 2019;23(8):5017-24.

37. Barker-Haliski M, White HS. Glutamatergic Mechanisms Associated with Seizures and Epilepsy. Cold Spring Harbor Perspect Med. 2015;5(8):a022863.

38. Clark IA, Vissel B. Excess cerebral TNF causing glutamate excitotoxicity rationalizes treatment of neurodegenerative diseases and neurogenic pain by anti-TNF agents. J Neuroinflammation. 2016;13(1):236.

39. Zhou Q, Viollet C, Efthymiou A, Khayrullina G, Moritz KE, Wilkerson MD, et al. Neuroinflammatory astrocytes generated from cord bloodderived human induced pluripotent stem cells. J Neuroinflammation. 2019;16(1):164

40. Diering GH, Huganir RL. The AMPA Receptor Code of Synaptic Plasticity. Neuron. 2018;100(2):314-29.

41. Widagdo J, Guntupalli S, Jang SE, Anggono V. Regulation of AMPA Receptor Trafficking by Protein Ubiquitination. Front Mol Neurosci. 2017;10:347.

42. Dohrke JN, Watson JF, Birchall K, Greger IH. Characterizing the binding and function of TARP $\gamma 8$-selective AMPA receptor modulators. J Biol Chem. 2020;295(43):14565-77.

43. Charsouei S, Jabalameli MR, Karimi-Moghadam A. Molecular insights into the role of AMPA receptors in the synaptic plasticity, pathogenesis and treatment of epilepsy: therapeutic potentials of perampanel and antisense oligonucleotide (ASO) technology. Acta Neurol Belg. 2020;120(3):531-44.

44. Itoh M, Yamashita M, Kaneko M, Okuno H, Abe M, Yamazaki M, et al. Deficiency of AMPAR-Palmitoylation Aggravates Seizure Susceptibility. J Neurosci. 2018;38(47):10220-35.
45. Wright SK, Wilson MA, Walsh R, Lo WB, Mundil N, Agrawal S, et al. Abolishing spontaneous epileptiform activity in human brain tissue through AMPA receptor inhibition. Ann Clin Transl Neurol. 2020;7(6):883-90.

46. French JA, Krauss GL, Biton V, Squillacote D, Yang H, Laurenza A, et al. Adjunctive perampanel for refractory partial-onset seizures: Randomized phase III study 304. Neurology. 2012;79(6):589-96.

47. AFJ, LKG, TWR, Xue-Feng W, Bree D, Christian B, et al. Perampanel for tonic-clonic seizures in idiopathic generalized epilepsy A randomized trial. Neurology. 2015;85(11):950-7.

48. Zaccara G, Giovannelli F, Cincotta M, ludice A. AMPA receptor inhibitors for the treatment of epilepsy: the role of perampanel. Expert Rev Neurother. 2013;13(6):647-55.

49. Liu T, Zhang L, Joo D, Sun SC. NF-kB signaling in inflammation. Signal Transduction Targeted Ther. 2017;2:17023.

50. Dresselhaus EC, Meffert MK. Cellular Specificity of NF-kB Function in the Nervous System. Front Immunol. 2019;10:1043.

51. Ting AT, Bertrand MJM. More to Life than NF-KB in TNFR1 Signaling. Trends Immunol. 2016;37(8):535-45.

52. JVAD, JMS, TK, RGD, MVI. Suppression of TNF-alpha-induced apoptosis by NF-kappaB. Science (New York, NY). 1996;274(5288):787-9.

53. Kim JE, Ryu HJ, Choi SY, Kang TC. Tumor necrosis factor-a-mediated threonine 435 phosphorylation of 065 nuclear factor-kB subunit in endothelial cells induces vasogenic edema and neutrophil infiltration in the rat piriform cortex following status epilepticus. J Neuroinflammation. 2012;9:6.

54. Xu J, Sun Y, Zhao Y, Li Q, Ge B, Dai S, et al. NF-kB "decoy" inhibits COX-2 expression in epileptic rat brain. J Integr Neurosci. 2020;19(1):77-87.

55. Toral-Rios D, Patiño-López G, Gómez-Lira G, Gutiérrez R, Becerril-Pérez F, Rosales-Córdova A, et al. Activation of STAT3 Regulates Reactive Astrogliosis and Neuronal Death Induced by A $\beta O$ Neurotoxicity. Int J Mol Sci. 2020;21(20):7458.

56. Li YZ, Zhang L, Liu Q, Bian HT, Cheng WJ. The effect of single nucleotide polymorphisms of STAT3 on epilepsy in children. Eur Rev Med Pharmacol Sci. 2020;24(2):837-42.

57. Wang H, Yao G, Li L, Ma Z, Chen J, Chen W. LncRNA-UCA1 inhibits the astrocyte activation in the temporal lobe epilepsy via regulating the JAK STAT signaling pathway. J Cell Biochem. 2020;121(10):4261-70.

58. Zhang X, Li X, Li B, Sun C, Zhang P. miR-21-5p protects hippocampal neurons of epileptic rats via inhibiting STAT3 expression. Adv Clin Exp Med. 2020;29(7):793-801.

59. Millot P, San C, Bennana E, Porte B, Vignal N, Hugon J, et al. STAT3 inhibition protects against neuroinflammation and BACE1 upregulation induced by systemic inflammation. Immunol Lett. 2020;228:129-34.

60. Chen E, Xu D, Lan X, Jia B, Sun L, Zheng JC, et al. A novel role of the STAT3 pathway in brain inflammation-induced human neural progenitor cell differentiation. Curr Mol Med. 2013;13(9):1474-84.

61. Ryu KY, Lee HJ, Woo H, Kang RJ, Han KM, Park H, et al. Dasatinib regulates LPS-induced microglial and astrocytic neuroinflammatory responses by inhibiting AKT/STAT3 signaling. J Neuroinflammation. 2019;16(1):190.

62. Pernice HF, Schieweck R, Kiebler MA, Popper B. mTOR and MAPK: from localized translation control to epilepsy. BMC Neurosci. 2016;17(1):73.

63. Huang Q, Liu X, Wu Y, Liao Y, Huang Y, Wei X, et al. P38 MAPK pathway mediates cognitive damage in pentylenetetrazole-induced epilepsy via apoptosis cascade. Epilepsy Res. 2017;133:89-92.

64. Ye Q, Zeng C, Luo C, Wu Y. Ferrostatin-1 mitigates cognitive impairment of epileptic rats by inhibiting P38 MAPK activation. Epilepsy Behav. 2020;103(Pt A):106670.

65. Wang C, Hong Z, Chen Y. Involvement of p38 MAPK in the Drug Resistance of Refractory Epilepsy Through the Regulation Multidrug Resistance-Associated Protein 1. Neurochem Res. 2015;40(7):1546-53.

66. Zhou X, Chen Q, Huang H, Zhang J, Wang J, Chen Y, et al. Inhibition of p38 MAPK regulates epileptic severity by decreasing expression levels of A1R and ENT1. Mol Med Rep. 2020;22(6):5348-57.

67. Zhang W, Wang X, Yu M, Li JA, Meng H. The c-Jun N-terminal kinase signaling pathway in epilepsy: activation, regulation, and therapeutics. J Recept Signal Transduct Res. 2018;38(5-6):492-8.

68. Chávez CE, Oyarzún JE, Avendaño BC, Mellado LA, Inostroza CA, Alvear TF, et al. The Opening of Connexin 43 Hemichannels Alters Hippocampal Astrocyte Function and Neuronal Survival in Prenatally LPS-Exposed Adult Offspring. Front Cell Neurosci. 2019;13:460. 
69. Zhang FF, Morioka N, Nakashima-Hisaoka K, Nakata Y. Spinal astrocytes stimulated by tumor necrosis factor-a and/or interferon- $\gamma$ attenuate connexin 43-gap junction via c-jun terminal kinase activity. J Neurosci Res. 2013;91(6):745-56.

70. Gómez Gl, Falcon RV, Maturana CJ, Labra VC, Salgado N, Rojas CA, et al. Heavy Alcohol Exposure Activates Astroglial Hemichannels and Pannexons in the Hippocampus of Adolescent Rats: Effects on Neuroinflammation and Astrocyte Arborization. Front Cell Neurosci. 2018;12:472.

- fast, convenient online submission

- thorough peer review by experienced researchers in your field

- rapid publication on acceptance

- support for research data, including large and complex data types

- gold Open Access which fosters wider collaboration and increased citations

- maximum visibility for your research: over 100M website views per year

At BMC, research is always in progress.

Learn more biomedcentral.com/submissions 